\title{
Impaired lamin localization to the nuclear envelope is responsible for nuclear damage in $L M N A$ mutant iPSC-derived cardiomyocytes
}

\author{
Maurer, Melanie $^{1,2}$, Perati, Shriya ${ }^{2}$, Johnson, Lindsey ${ }^{2},{\text { Gacita, Anthony } \mathrm{M}^{3} \text {, Lai, Shuping }}^{4}$, \\ Wallrath, Lori L ${ }^{5}$, Benjamin, Ivor J ${ }^{4}$, McNally, Elizabeth M. $^{3}$, Kirby, Tyler J ${ }^{2,6}$ Lammerding, \\ $\operatorname{Jan}^{1,2, *}$
}

${ }^{1}$ Meinig School of Biomedical Engineering, Cornell University, Ithaca, NY 14850; ${ }^{2}$ Weill Institute for Cell and Molecular Biology, Ithaca, NY 14850; ${ }^{3}$ Center for Genetic Medicine, Feinberg School of Medicine, Northwestern Medicine, Chicago, IL 60611; ${ }^{4}$ Cardiovascular Center, Medical College of Wisconsin, Milwaukee, WI 53226; ${ }^{5}$ Department of Biochemistry and Molecular Biology, University of Iowa, Iowa City, IA 52242; ${ }^{6}$ Department of Physiology, Amsterdam UMC Location VUmc, Amsterdam, The Netherlands

*, correspondence should be directed at jan.lammerding@,cornell.edu

\begin{abstract}
The $L M N A$ gene encodes the nuclear envelope proteins Lamins A and C, which comprise a major part of the nuclear lamina, provide mechanical support to the nucleus, and participate in diverse intracellular signaling. $L M N A$ mutations give rise to a collection of diseases called laminopathies, including dilated cardiomyopathy (LMNA-DCM) and muscular dystrophies. Although nuclear deformities are a hallmark of $L M N A$-DCM, the role of nuclear abnormalities in the pathogenesis of $L M N A$-DCM remains incompletely understood. Using induced pluripotent stem cell-derived cardiomyocytes (iPSC-CMs) from $L M N A$ mutant patients and healthy controls, we show that $L M N A$ mutant iPSC-CM nuclei have altered shape or increased size compared to healthy control iPSC-CM nuclei. The $L M N A$ mutation exhibiting the most severe nuclear deformities, R249Q, additionally caused reduced nuclear stiffness and increased nuclear fragility. Importantly, for all cell lines, the degree of nuclear abnormalities corresponded to the degree of Lamin A/C and Lamin B1 mislocalization from the nuclear envelope. The mislocalization was likely due to altered assembly of Lamin A/C. Collectively, these results point to the importance of correct lamin assembly at the nuclear envelope in providing mechanical stability to the nucleus and illustrate that defects in nuclear lamina organization can contribute to the nuclear and cellular dysfunction in $L M N A$-DCM.
\end{abstract}




\section{Introduction}

Lamin $\mathrm{A} / \mathrm{C}$ are intermediate filaments that assemble to form the nuclear lamina, a dense protein meshwork that resides underneath the inner nuclear membrane. The nuclear lamina provides structural support to the nucleus and functions in diverse mechanical and biochemical signaling processes (Davidson and Lammerding, 2014; Kirby and Lammerding, 2018; Maurer and Lammerding, 2019; Donnaloja et al., 2020). More than 450 mutations have been identified in the $L M N A$ gene that give rise to a collection of about 15 different human diseases, termed 'laminopathies,' which include dilated cardiomyopathy ( $L M N A$-DCM), Emery-Dreifuss muscular dystrophy (EDMD), congenital muscular dystrophy (CMD), and Hutchinson-Gilford progeria syndrome (HGPS) (Davidson and Lammerding, 2014). LMNA-DCM has a high mortality rate, and compared to other forms of congenital DCM, LMNA-DCM has a particularly poor prognosis with early onset, a high occurrence of arrhythmias and up to $19 \%$ of all patients requiring heart transplants (Taylor et al., 2003; McNally and Mestroni, 2017; Hasselberg et al., 2018). To date, clinical treatments for $L M N A$-DCM have focused on slowing heart failure rather than targeting the underlying cellular pathology, largely because the molecular disease mechanisms responsible for $L M N A$-DCM are not yet fully understood.

$L M N A$ mutations and deletions often cause a reduction in nuclear stiffness that results in changes to nuclear shape, nuclear envelope (NE) rupture, and altered response to mechanical stress (Broers et al., 2004; Lammerding et al., 2004; Nikolova et al., 2004; Chandar et al., 2010; De Vos et al., 2011; Zwerger et al., 2013; Earle et al., 2019; Bertrand et al., 2020). However, few studies have been completed to understand nuclear mechanics and nuclear deformation in LMNA mutant human cardiomyocytes. Until the recent advent of human induced pluripotent stem cell (iPSC) models, the majority of studies on the cellular disease pathogenesis of $L M N A$-DCM have relied on mouse models that do not fully reflect all aspects of the human disease. For example, mutant mouse phenotypes require homozygous mutant alleles rather than dominant heterozygous allelic state in human disease (Stewart et al., 2007). As such, limited previous studies have demonstrated nuclear shape changes and NE ruptures resulting in the mislocalization of cellular components into the nucleus in cardiomyocytes of laminopathy models (Nikolova et al., 2004; Chandar et al., 2010; Cho et al., 2019; Shah et al., 2019). Despite these efforts, the cardiac-specific mechanisms through which nuclei become damaged, the consequences of nuclear damage, and the effect of specific $L M N A$ mutations in the context of nuclear damage in cardiomyocytes remain largely unclear. Since the contractile behavior of cardiomyocytes can influence nuclear mechanical properties through lamin conformation (Swift et al., 2013; Buxboim et al., 2014; Heffler et al., 2019; Piccus and Brayson, 2020), it is critical to determine the cardiomyocyte-specific role of nuclear mechanics and damage in $L M N A$-DCM and how they contribute to disease progression.

Here, we use three $L M N A$ mutant patient-derived human iPSC lines, iPSCs from healthy controls, and iPSC-derived cardiomyocytes (iPSC-CMs) to provide a quantitative analysis of the causes and consequences of nuclear damage in $L M N A$-DCM, with a particular focus on the nuclear lamina organization and physical properties of the cell nucleus. We show that different $L M N A$ mutant iPSC-CMs exhibit varying degrees of nuclear shape and size deformities, with only cells with the most severe mutation, LMNA R249Q, exhibiting NE rupture. Furthermore, we demonstrate that the severity of nuclear deformities correlates with reduced Lamin A/C and Lamin $\mathrm{B} 1$ localization to the NE, likely due to defective lamin assembly. Assembly of Lamin A/C at the $\mathrm{NE}$ is critical for maintaining nuclear mechanical properties and NE integrity (Zwerger et al., 2015). As such, defective assembly of lamins at the NE may cause reduced nuclear stability and/or defects in organization of NE proteins that in turn may result in altered cytoskeletal organization, 
abnormal nuclear shape, and susceptibility of the nucleus to damage. Collectively, these findings suggest that mislocalization of Lamin $\mathrm{A} / \mathrm{C}$ and Lamin B1 from the NE can explain much of the nuclear deformities and damage observed in three $L M N A$-DCM iPSC-CM lines.

\section{Materials and Methods}

iPSC culture and cardiac differentiation

Healthy control (WT) and patient-derived LMNA mutant iPSCs were either purchased from commercial sources (Cure CMD) or generously provided from other researchers (Fig. 1A; Table 1). iPSCs were cultured feeder-free in TeSR-E8 media (\#05990, StemCell Technologies) on 1:30 Matrigel Basement Membrane (Matrigel; \#47743-722, Corning) diluted in RPMI 1640 medium (\#11875093, Gibco). Media was changed daily, and colonies were monitored for and removed with signs of spontaneous differentiation.

Cardiac differentiation was performed based on a previously established protocol (Sharma et al., 2015). iPSCs were washed with RMPI 1640 medium, dissociated into single cells with TrypLE Express (\#12605028, Gibco), and seeded on 1:30 Matrigel in 12-well plates in TeSR-E8 media supplemented with Y-27632 (10 $\mu \mathrm{M}$, \#10005583, Cayman Chemical). Media was changed to fresh TeSR-E8 after 24 hours. After 48 hours (D0), differentiation was induced using inhibition of the WNT pathway with CHIR99021 (6 $\mu \mathrm{M}$ or $12 \mu \mathrm{M}$, \#SML1046, Sigma-Aldrich) in RPMI 1640 media supplemented with B27 minus insulin (\#A1895602, Gibco; referred to here as B27INS). The CHIR99021 concentration, at $6 \mu \mathrm{M}$ or $12 \mu \mathrm{M}$, was optimized for each cell line as each had different sensitivities to the inhibitor. Media was changed to fresh B27-INS at day 1 of differentiation (D1). IWR-1 (5 $\mu$ M, \#13659, Cayman Chemical) in B27-INS was added at D3 and changed to fresh B27-INS at D5. Media was changed to RPMI 1640 medium supplemented with B27 (\#17504044, Gibco; referred to as B27) on D7 and was changed daily for three days until beating was induced. On D10, media was changed to RPMI 1640 without L-Glutamine (\#21870076, Gibco; referred to as selection medium) supplemented with B27 and Sodium Lactate (Sigma) for metabolic selection of cardiomyocytes. On D12, media was changed to B27 supplemented with Penicillin-Streptomycin (referred to as B27+P/S), and iPSC-CMs were allowed to recover for two days before passaging ('first CM passage').

At D14, only wells of iPSC-CMs that were beating were washed with RPMI 1640 and dissociated into single cells using Trypsin (0.25\%). Trypsin was inactivated using RPMI 1640 plus $20 \%$ fetal bovine serum. Cells were centrifuged and resuspended in RPMI 1640 plus fetal bovine serum $(10 \%)$ and $10 \mu \mathrm{M}$ Y-27632 and then seeded 1:1 in 12-well plates coated with Fibronectin $(4 \mu \mathrm{M})$. After 24 hours, media was changed to B27+P/S. After an additional 24 hours, iPSC-CMs were subjected to a second round of metabolic selection with selection medium for 48 hours. Media was then changed back to $\mathrm{B} 27+\mathrm{P} / \mathrm{S}$, and cells were used seven days after the first $\mathrm{CM}$ passage (D21).

After 21 days of differentiation, all cell lines spontaneously contracted throughout the wells. We confirmed cardiac differentiation by immunofluorescence staining for a cardiac marker, cardiac troponin T (cTnT; Fig. 1B). We quantified the percentage of cells that expressed cTnT, which demonstrated that all cell lines routinely had over $85 \%$ expression of cTnT and no significant changes in expression between cell lines (Fig. 1C). All immunofluorescence experiments were performed with a cTnT co-stain. Upon immunofluorescence labeling, only wells with predominantly cTnT-expressing cells were used for analysis (wells with few cTnT-expressing cells were excluded), and any remaining individual cells not expressing cTnT were excluded from analysis. 
Generation of fluorescently labeled cell lines

iPSC-CMs after the first CM passage were stably modified with lentiviral constructs to express a NE rupture reporter consisting of a green fluorescent protein with a nuclear localization sequence (NLS-GFP, full vector: pCDH-CMV-NLS-copGFP-EF1-blastiS) described previously (Denais et al., 2016). Cells were cultured for at least six days after modification to allow sufficient expression of the construct. Expression of NLS-GFP was confirmed before each experiment.

\section{Lamin A/C depletion}

Prior to the first round of metabolic selection, WT2-iPSC-CMs were stably modified with a lentivirus to express either shRNA targeting LMNA (shLMNA; pLKO.1-shRNA-mLMNA; target sequence: CCGGGAAGCAACTTCAGGATGAGATCTCGAGATCTCATCCTGAAGTTGCTTCTTTTT G) or a non-target control (shNT). For all experiments, shRNA-modified iPSC-CMs were used as a mixed population of modified ('knockdown' or 'KD') and un-modified ('no knockdown' or 'no KD') cells to serve as an additional internal control group, as described in the Image Analysis section.

\section{Long-term imaging experiments}

Long-term fluorescence imaging of iPSC-CMs expressing NLS-GFP imaging was performed on an IncuCyte (Sartorius) incubator imaging system. Images were acquired at $10 \times$ magnification every 15 minutes for three days, starting one week after the first CM passage. Images were exported and manually analyzed in FIJI software to quantify the frequency and duration of NE rupture, as evidenced by the transient loss of NLS-GFP from the nucleus.

\section{Immunofluorescence staining}

iPSC-CMs were passaged one week after the first CM passage into optically clear 96-well plates. The next day, media was changed to B27+P/S for several hours before cells were washed with $1 \times$ PBS and fixed in warm Paraformaldehyde (4\%) for 10 minutes. Cells were then washed with $1 \times$ PBS and blocked in 3\% BSA with $0.1 \%$ Triton-X 100 (Thermo-Fisher) and $0.1 \%$ Tween (Sigma) in PBS for one hour at room temperature. Primary antibodies (Table S1) were prepared in blocking solution and incubated overnight at $4{ }^{\circ} \mathrm{C}$. iPSC-CMs were then washed with a solution of $0.3 \%$ BSA with $0.1 \%$ Triton-X 100 and $0.1 \%$ Tween in PBS and stained with AlexaFluor secondary antibodies (1:250, Invitrogen) for 1 hour at room temperature. DAPI (1:1000, Sigma) was added for 15 minutes at room temperature, and cells were washed with PBS before imaging.

\section{Nuclear semi-permeabilization and soluble protein washout}

One week after the first CM passage, iPSC-CMs were passaged into optically clear 96-well plates (Greiner). The next day, media was changed to B27+P/S for several hours before cells were washed with $1 \times$ PBS. A buffer, "CSKT," containing $\mathrm{NaCl}(100 \mathrm{mM})$, Sucrose $(300 \mathrm{mM}), \mathrm{MgCl}_{2}$ (3 mM), PIPES (10 mM, pH 6.8) and Triton-X 100 (0.5\%) was added to cells to semi-permeabilize nuclei and incubated on ice for 1 minute. A 1 -minute wash with $1 \times$ PBS was used as a control in parallel to semi-permeabilization with CSKT. CSKT buffer was removed, cells were gently washed with $1 \times$ PBS to washout soluble nuclear proteins, and then the cells were fixed with $4 \%$ PFA for 10 minutes. Following PFA fixation, cells were inspected under a microscope to ensure 
that nuclear semi-permeabilization and washout was successful, as evidenced by the darkened and more prominent appearance of nuclei.

\section{Image acquisition}

Plates were imaged on an inverted Zeiss LSM700 confocal microscope. Z-stacks were taken using a $40 \times$ water immersion (1.2 NA) objective with Airy units set to 1.0 for all images. Zstacks for nuclear volume were acquired using the optimum step size for the $40 \times$ water immersion objective, i.e., $0.35 \mu \mathrm{m}$. All other image stacks were acquired using a step size of $1.5 \mu \mathrm{m}$ and analyzed using Maximum Intensity Projections (MIPs).

\section{Image analysis}

All image analysis was conducted by observers blinded for genotype and treatment conditions. iPSC-CM purity was quantified from MIPs by counting the proportion of nuclei in the image that are located within cardiac troponin $\mathrm{T}(\mathrm{cTnT})$ positive cells (referred to as cTnT-positive nuclei). For image analysis of iPSC-CMs, only cTnT-positive nuclei were used.

For nuclear shape and area, a custom MATLAB (MathWorks) script was used to segment nuclei based on the Lamin B1 immunofluorescence signal in MIPs. Nuclei intersecting with the image edges were removed, followed by manual selection of nuclei in each image to exclude any nuclei touching each other, dead cells, or non-cTnT-positive nuclei. The area (A) and perimeter (P) were then measured for each selected nucleus, and the circularity index (CI) was computed based off the formula $C I=\frac{4 \pi A}{P^{2}}$. A perfectly circular object has a circularity index of 1 , and the circularity index decreases as circularity decrease, e.g., in oval or irregularly shaped nuclei.

Nuclear volume was computed from high resolution confocal Lamin B1 immunofluorescence 3D image stacks. Image stacks were read into the FIJI image analysis program, and a threshold value was determined from the middle slice of z-stacks. The threshold value was then input into the 3D Simple Segmentation suite (Ollion et al., 2013), and the 3D Objects Counter (Bolte and Cordelières, 2006) was used to compute nuclear volumes. Partial nuclei at the image edges, nuclei touching each other, dead cells, and non-cTnT-positive nuclei were excluded from analysis.

For shRNA experiments, nuclear area, circularity index, and Lamin A/C mean fluorescence intensity were computed from a modified version of the MATLAB script for nuclear area and circularity described above. Nuclear area and circularity index were computed from the Lamin B1 immunofluorescence signal of MIPs as described above. Additionally, Lamin A/C images were read into the program, images were cross-correlated with the corresponding Lamin B1 images to analyze only the user-selected nuclei, and the mean Lamin $\mathrm{A} / \mathrm{C}$ immunofluorescence intensity inside of the area of each user-selected nucleus was computed. For each individual experiment, Lamin A/C intensities for shNT- and shLMNA-treated cells were plotted, and the lowest Lamin $\mathrm{A} / \mathrm{C}$ intensity from the shNT group was used as a threshold, above which shLMNA nuclei were considered "no knockdown (no KD)" and below which shLMNA nuclei were considered "knock down (KD)." shLMNA nuclei were separated into "no KD" or "KD” groups for each experiment, with "no KD" nuclei serving as a secondary, internal control. Nuclear area and circularity index were analyzed based on the average of several separately thresholded experiments.

Nuclear volume analysis of shRNA Lamin A/C depleted cells and controls was performed using the same FIJI image analysis pipeline described above. For these experiments, an observer blinded for treatment conditions manually classified nuclei as "no KD" or "KD" for each image based on their Lamin A/C immunofluorescence intensity. Untreated control or shNT groups, as 
expected, had extremely few nuclei considered as "KD" and the proportion of shLMNA no KD and KD nuclei was similar to that obtained by the objective thresholding method.

Fluorescence intensity profiles were computed from high-resolution confocal z-stacks in the ZEN software (Zeiss). For each nucleus, the z-position for which the $\mathrm{x}-\mathrm{y}$ plane dissects the center of the nucleus was identified, and a line was drawn across the major axis of the nucleus to obtain the Lamin A/C and Lamin B1 fluorescence intensity values along with distances. Fluorescence intensity profiles were trimmed such that the first values in the profile correspond to the outer edges of the NE. Distances across each nucleus were then normalized to a scale of 0 to 1 to account for differences in nuclear size. Fluorescence intensity profiles and normalized distances for each nucleus for each cell line were then read into a custom $\mathrm{R}$ script for analysis (script available upon request). Fluorescence intensity profiles across all nuclei in a cell line were averaged and normalized to the area under the curve to account for variations in fluorescence intensities between cell lines due to varying lamin levels or staining conditions. The boundary of the nuclear lamina was determined by the inflection point of the average fluorescence intensity profile of all cell lines with the nuclear lamina defined as the peripheral regions with normalized nuclear distance of $<0.16$ and $>0.84$. For each nucleus, the fluorescence intensity of nucleoplasmic lamins was then determined by taking the $95^{\text {th }}$ percentile of fluorescence intensity values in the nuclear lamina region (to avoid influence of spurious pixel intensity values), and the fluorescence intensity of nucleoplasmic lamins was determined by taking the average value of fluorescence intensities in the nucleoplasm, corresponding to normalized nuclear distances between 0.16 and 0.84. The ratio of nucleoplasmic to peripheral lamins was then computed for each nucleus.

Lamin $\mathrm{A} / \mathrm{C}$ and phospho-Lamin $\mathrm{A} / \mathrm{C}$ fluorescence intensities were computed using the same MATLAB script used for shRNA analysis described above with Lamin A/C images being used for thresholding and user selection under the same exclusion criteria as previously selected. All Lamin A/C and phospho-Lamin A/C average fluorescence intensity values were normalized to the respective average of a healthy control (WT1) fluorescence intensity from images taken at the same time.

\section{Nuclear stiffness measurements}

iPSC-CMs were passaged one week after the first CM passage into glass-bottom 35-mm tissue culture plates (FluoroDish; World Precision Instruments) coated with $4 \mu \mathrm{M}$ Fibronectin and allowed to adhere for 24 hours. Prior to experimentation, nuclei were fluorescently labeled with Hoechst 33342 (1:1000; Biotium). Experiments were performed at room temperature, and all indentations were performed within one hour of removing cells from the incubator, at which point the majority of cells were still beating.

Nanoindentation was performed with a microscope-mounted Chiaro system (Optics11). The system was calibrated prior to experimentation according to the manufacturer's instructions, and a spherical glass tip with a radius of $3 \mu \mathrm{m}$ and stiffness of $0.026 \mathrm{~N} / \mathrm{m}$ was used to indent cells. The center of the probe tip and the center of nuclei were aligned, and an image of each fluorescent nucleus was taken prior to indentation. The probe was then lowered to approximately 5-10 $\mu \mathrm{m}$ above the top of the nucleus. If the probe accidentally touched a nucleus prior to the start of the indentation, the cell was excluded. The probe was lowered towards the nucleus at a speed of 2 $\mu \mathrm{m} / \mathrm{s}$, and once the system reached a contact force threshold of $5 \mathrm{nN}$, indentation continued at a rate of $10 \mathrm{nN} / \mathrm{s}$ until the probe reached a force threshold of $40 \mathrm{nN}$. The $40 \mathrm{nN}$ load was held for five seconds, and then the probe was retracted at a rate of $10 \mathrm{nN} / \mathrm{s}$. A Hertzian model with a Poisson's Ratio of 0.5 (Guz et al., 2014) was fitted to the load vs. indentation curve from 250- 
$1500 \mathrm{~nm}$ of indentation depth for each nucleus to determine the Young's elastic modulus. Any nucleus with a fit for the Hertzian model below an $\mathrm{R}^{2}$ value of 0.95 was excluded from further analysis.

\section{Western analysis}

iPSC-CMs lysates were collected one week after the initial passage. Cells were lysed in High Salt RIPA buffer with protease (cOmplete EDTA-Free, Roche) and phosphatase inhibitors (PhosSTOP, Roche). After lysis, samples were vortexed for five minutes, sonicated for 30 seconds at $36 \%$ amplitude, and boiled for five minutes at $93^{\circ} \mathrm{C}$. Protein content was quantified with BioRad Protein Assay Dye, and $20 \mu \mathrm{g}$ of protein was run on a 4-12\% Bis-Tris polyacrylamide gel using an SDS-PAGE protocol. Protein was transferred to a polyvinylidene fluoride (PVDF) membrane using a semi-dry transfer at $16 \mathrm{~V}$ for one hour. Membranes were blocked with Intercept (PBS) Blocking Buffer (LI-COR) for one hour at room temperature, primary antibodies were diluted in the same blocking buffer, and membranes were incubated the primary antibodies on a rocker overnight at $4^{\circ} \mathrm{C}$. Loading control primary antibodies were diluted in the same blocking buffer and incubated for one hour at room temperature the next day. IRDye 680LT or IRDye $800 \mathrm{CW}$ (LI-COR) secondary antibodies were used to detect protein bands. Membranes were imaged on an Odyssey ${ }^{\circledR}$ CLx imaging system (LI-COR) and analyzed using Image Studio Lite (LI-COR).

\section{Gene expression analysis}

RNA was extracted from iPSCs before passaging when colonies were large but not touching and iPSC-CMs one week after their initial passage using the RNeasy Plus Kit (Qiagen). Quality of the RNA was assessed using a Fragment Analyzer. Libraries were prepared by the Cornell Institute of Biotechnology using a TruSeq DNA library prep kit (Illumina) and sequenced on a NextSeq 500/550 (Illumina). Raw sequencing reads were quality checked using FastQC (Andrew, 2010). STAR (Dobin et al., 2013) was used to assemble the human genome and align reads to the human genome. Aligned reads were quality control checked, and samples with alignment $<70 \%$ were removed. A count matrix was generated using featureCounts (Liao et al., 2014) which was then input to build a statistical model in DESeq2 (Love et al., 2014). Normalized gene counts were then extracted for each sample for LMNA and LMNB1.

\section{Code availability}

Custom MATLAB scripts utilized here are available upon request.

\section{Statistical analysis}

All results were taken from a minimum of three independent experiments. For continuous numeric datasets in which individual nuclei were analyzed, a mixed-effects linear regression was performed in $\mathrm{R}$ to account for the effects of both $L M N A$-mutations and individual cell lines. Datasets were tested for normality, and data not following a normal distribution were linearized either by taking the natural log or the square root, whichever achieved better normalization. Pairwise $t$-tests were then performed using the Tukey method to correct for multiple comparisons. For continuous numeric datasets in which whole images or experiments were analyzed and followed a normal distribution, either a Student's $t$-test (for two groups) or one-way analysis of variance (ANOVA) with multiple comparisons (for more than two groups) was performed in Prism (GraphPad). Dunnett correction was used for multiple comparisons. For NE rupture experiments 
in which proportional data was analyzed, a binomial regression was performed in R. Since several cell lines exhibited no NE rupture, a Firth logistic regression was then performed to reduce bias from such zero values. Pairwise $t$-tests were then performed using the Tukey method to correct for multiple comparisons. For correlation data, a linear regression was performed for each cell line in Graphpad Prism, and the correlation was tested for significance for a non-zero slope using Pearson correlation and compared to the other regression. Unless otherwise noted, error bars in graphs represent mean \pm standard error of the mean (SEM).

\section{Results}

LMNA mutant iPSCs differentiate efficiently into cardiomyocytes

To quantitatively study the effects of different $L M N A$ mutations on nuclear lamina organization and nuclear mechanics, we selected three $L M N A$ mutant iPSC lines (LMNA L35P, R249Q, and G449V) derived from patients with DCM and CMD and two healthy controls (WT1, WT2) for this study (Table 1). Each of the $L M N A$ mutations cause amino acid substitutions in a different domain of the Lamin A/C protein (Fig. 1A), enabling the identification of common and mutation-specific effects. The iPSCs were subjected to a chemically defined cardiac differentiation protocol to obtain iPSC-derived cardiomyocytes (iPSC-CMs). All iPSCs underwent successful cardiac differentiation with over $85 \%$ of nuclei staining positive for cardiac troponin $\mathrm{T}$ (cTnT), an established cardiomyocyte marker (Fig. 1B, C). We did not detect any significant differences in iPSC-CM differentiation efficiency between cell lines (Fig. 1C).

\section{LMNA mutant iPSC-CMs express normal levels of Lamin A/C}

Although most $L M N A$ mutations result in stable expression of the mutant protein, some LMNA mutations can reduce protein stability and lead to haploinsufficiency (Wolf et al., 2008; Siu et al., 2012; Cattin et al., 2013). To test whether the LMNA mutant cell lines had altered expression of Lamin $\mathrm{A} / \mathrm{C}$, we investigated protein and mRNA expression levels of Lamin $\mathrm{A} / \mathrm{C}$ and Lamin B1 in the panel of iPSCs and the corresponding iPSC-CMs. We detected only very low Lamin A/C protein levels in iPSCs by both western analysis (Fig. 2A) and immunofluorescence labeling (Fig. S1A), consistent with previous reports that iPSCs express no or only little Lamin A/C (Zuo et al., 2012). As expected, Lamin A/C expression increased significantly with differentiation into iPSCCMs (Fig. 2A, Fig. S2B). Differences in Lamin A/C expression were not significant between the various iPSC-CMs, although the R249Q mutant iPSC-CMs showed a trend towards reduced Lamin A/C protein expression ( $p>0.15 v s$. both healthy control lines) (Fig. 2A-B, Fig. S2C-D). Lamin B1 expression did not differ significantly between iPSC-CMs cell lines (Fig. 2A-B), although the R249Q iPSCs had a trend towards increased Lamin B1 mRNA levels compared to ( $p>0.14$ vs. both healthy control lines) (Fig. S2B).

\section{LMNA mutant iPSC-CMs exhibit altered nuclear shape and size}

Although altered nuclear shape and size are hallmarks of skeletal muscle and cardiac laminopathies, the severity of nuclear shape defects varies substantially across different $L M N A$ mutations (Sullivan et al., 1999; Lammerding et al., 2004, 2006; Muchir et al., 2004; Zwerger et al., 2013; Steele-Stallard et al., 2018; Bertrand et al., 2020). To quantify defects in nuclear morphology in the different LMNA mutant iPSC-CMs, we computed nuclear circularity index, area, and volume based on confocal 3D image stacks and cross-sections of iPSC-CMs immunofluorescently labeled for Lamin B1 (Fig. 3A). The circularity index has a maximum value of one for perfectly circular nuclei and decreases in value for abnormally shaped nuclei (Fig. 3B). 
R249Q mutant iPSC-CMs had a significantly decreased circularity index compared to healthy controls and other $L M N A$ mutants (Fig. 3C), indicating more abnormal nuclear shapes. In contrast, G449V mutant iPSC-CM nuclei exhibited no change in circularity index compared to healthy controls, and L35P mutant nuclei were slightly more circular compared to healthy controls and the other mutant iPSC-CMs (Fig. 3C). Notably, all three LMNA mutant iPSC-CM lines had increased nuclear cross-sectional areas compared to healthy controls (Fig. 3D), although only R249Q and L35P mutant iPSC-CMs showed reduction in nuclear height (Fig. 3E) and increased nuclear volumes (Fig. 3F) compared to healthy controls. These data suggest more severe nuclear defects in L35P and R249Q mutant iPSC-CMs with increased nuclear area and volume, potentially pointing to changes in nuclear organization and/or nuclear stiffness, as decreased nuclear height and increased nuclear cross-sectional area can result in increased nuclear flattening under cytoskeletal tension.

\section{R249Q-iPSC-CMs exhibit increased nuclear envelope rupture}

In addition to changes to nuclear morphology, $L M N A$ mutations can lead to increased incidence of nuclear envelope (NE) rupture due to weakening of the NE (De Vos et al., 2011; Cho et al., 2019; Earle et al., 2019). Therefore, we modified iPSC-CMs with a NE rupture reporter, NLS-GFP, in which a green fluorescent protein (GFP) is fused to a nuclear localization sequence (NLS) (Denais et al., 2016). NLS-GFP normally localizes to the nucleus but leaks into the cytoplasm upon NE rupture and then gradually translocates back into the nucleus following NE repair (Fig. 4A-B). Long-term (72 hours) time-lapse microscopy revealed that NE rupture is extremely rare in healthy control iPSC-CMs (Fig. 4C). In contrast, R249Q mutant iPSC-CMs, which had the most severe defects in nuclear shape, size, and volume (Fig. 3C-E), had a significant increase of NE ruptures compared to both healthy control cell lines (Fig. 4C). On the other hand, the L35P and G449V mutant iPSC-CMs, which had milder defects in nuclear shape and size, showed no increase in NE rupture or only a trend towards increased NE rupture (G449V) that did not reach statistical significance ( $p>0.3$ vs healthy controls). Of note, the duration of NE rupture was not statistically significant between any of the cell lines that exhibited NE rupture (Fig. S3), suggesting that $L M N A$ mutations did not alter NE repair, consistent with previous studies that found that Lamin A/C depletion did not alter NE rupture duration (Denais et al., 2016; Halfmann et al., 2019). The dramatic increase of NE rupture in the R249Q mutant iPSC-CMs compared to other LMNA mutant cell lines, paired with the R249Q iPSC-CMs being the only cell line to have a reduction in circularity index, suggests that R249Q mutant iPSC-CMs have more dramatic changes in nuclear stability, potentially due to changes in nuclear mechanics, that leave the nucleus more susceptible to damage.

\section{R249Q-iPSC-CMs have reduced nuclear stiffness}

Lamin A/C are a major determinant of nuclear stiffness (Lammerding et al., 2004, 2006; Swift et al., 2013; Stephens et al., 2017), and LMNA-mutations can cause changes in nuclear stability that lead to nuclear damage (Sullivan et al., 1999; Nikolova et al., 2004; Gupta et al., 2010; De Vos et al., 2011; Zwerger et al., 2013; Cho et al., 2019; Earle et al., 2019). To determine whether the high degree of nuclear defects exhibited by R249Q mutant nuclei could be related to altered nuclear mechanics, we measured nuclear stiffness of R249Q mutant iPSC-CMs and healthy controls with a Chiaro Nanoindenter (Optics11; Fig. 4D). We confirmed that nuclei in iPSC-CMs are close to the cell surface with little to no cytoskeleton over the top of nuclei (Fig. S4A). Thus, when probing the nucleus in intact cells, the resulting force-indentation curves are expected to 
primarily reflect the mechanical properties of the cell nucleus. The R249Q iPSC-CMs had significantly reduced nuclear stiffness (Fig. 4E) which was also reflected by the fact that at the same indentation force, the R249Q mutant iPSC-CM nuclei had a significantly increased indentation depth compared to healthy control iPSC-CMs (Fig. S4B). Interestingly, although R249Q mutant iPSC-CMs had increased nuclear cross-sectional areas compared to healthy control iPSC-CMs (Fig. S4D), consistent with our earlier measurements (Fig. 2D), we did not find a significant correlation between nuclear cross-sectional area and nuclear stiffness across individual cells for either cell line (Fig. S4B), likely due to the large variability in nuclear cross-sectional area between individual cells.

\section{Reduced Lamin A/C expression can partially explain R249Q nuclear damage}

Since the iPSC-CM lines have a heterogeneous genetic background, we wanted to confirm whether the defects in nuclear shape and stability observed in the R249Q mutant iPSC-CMs could be attributed to the reduced levels or function of Lamin $\mathrm{A} / \mathrm{C}$ levels in these cells. To do so, we assessed the effect of shRNA-mediated Lamin A/C depletion (shLMNA) in healthy control iPSCCMs on nuclear morphology and NE rupture. Cells treated with non-target shRNA (shNT) served as isogenic controls. Depletion of Lamin A/C was confirmed by immunofluorescence labeling. Due to limited transduction efficiency in iPSC-CMs, only about 50\% of the shLMNA-modified cells exhibited loss of Lamin A/C ('shLMNA-KD' cells) at 7 days post transfection, with the remainder showing near normal Lamin A/C levels ('shLMNA-no KD' cells) (Fig. 5A; Fig. S5AB). The shLMNA-KD iPSC-CMs had significantly increased nuclear area and volume compared to shLMNA-no KD and shNT controls (Fig. 5C-D), consistent with the increase in nuclear area and volume observed in the R249Q cells (Fig. 3D-E). Surprisingly, we did not detect any changes in circularity index between shLMNA-KD nuclei and either shNT or shLMNA-no KD nuclei (Fig. 5B). These findings suggest that reduced Lamin A/C levels directly influence nuclear size in iPSCCM nuclei, but other factors may contribute to abnormal nuclear shape, although it is also possible that prolonged loss of Lamin $\mathrm{A} / \mathrm{C}$ is required to cause more substantial defects in nuclear shape or the timing of Lamin A/C loss during cardiac differentiation of iPSC-CMs influences nuclear shape.

Time-lapse experiments with shLMNA- and shNT-modified iPSC-CMs expressing the NLS-GFP NE rupture reporter suggested that Lamin A/C depletion may increase NE rupture (Fig. $5 \mathrm{E}$ ), although the difference between the shLMNA iPSC-CMs and shNT controls was not quite statistically significant $(p=0.12)$. This lack of statistical significance was likely due to the limited transduction efficiency, in which only $\sim 50 \%$ of the observed shLMNA cells are expected to actually lack Lamin A/C (Fig. S5B). Overall, these data are consistent with a previous study reporting increased $\mathrm{NE}$ rupture in Lamin A/C depleted cardiac myocytes (Cho et al., 2019), and suggest that reduced Lamin A/C levels or functional loss of Lamin A/C could be at least partially responsible for the increase in NE ruptures in the R249Q mutant iPSC-CMs. On the other hand, the fraction of shLMNA iPSC-CMs exhibiting NE rupture was substantially lower than in the R249Q mutant cells, even when accounting for the reduced transduction efficiency, suggesting that additional mechanism contribute to the increased incidence of NE rupture in the R249Q mutant iPSC-CMs.

\section{LMNA mutant iPSC-CMs exhibit reduced localization of lamins to the nuclear envelope}

$L M N A$ mutations may alter Lamin $\mathrm{A} / \mathrm{C}$ self-assembly or lamin phosphorylation, resulting in improper assembly of the lamina and mislocalization of Lamin $\mathrm{A} / \mathrm{C}$ from the $\mathrm{NE}$ (Cenni et al., 2005; Wiesel et al., 2008; Zwerger et al., 2015; Bertrand et al., 2020). Since defects in lamin 
assembly could impair the resistance of the nucleus to mechanical forces exerted by the cytoskeleton, resulting in nuclear defects and NE rupture (De Vos et al., 2011; Zwerger et al., 2013; Denais et al., 2016), we examined the intranuclear distribution of Lamin A/C and Lamin B1 in the LMNA mutant and healthy control iPSC-CMs by immunofluorescence labeling. Both the R249Q and the L35P mutant iPSC-CMs frequently had reduced Lamin A/C fluorescence at the nuclear periphery (Fig. 6A). For a more quantitative analysis, we measured the immunofluorescence intensity profiles of Lamin A/C (Fig. 6B) and Lamin B1 (Fig. S6) along a line across the central z-plane of nuclei. Lamin fluorescence intensity profiles were normalized to the nuclear diameter and to the area under the curve to account for variations in nuclear size and Lamin A/C levels (Fig. 6C). Whereas healthy control iPSC-CMs exhibited Lamin A/C fluorescence intensity profiles with large peaks at the nuclear periphery, indicating that Lamin $\mathrm{A} / \mathrm{C}$ is predominantly found at the nuclear lamina in these cells, the R249Q, L35P, and, to a lesser extent, G449V mutant iPSC-CMs had significantly decreased Lamin A/C at the nuclear periphery and increased Lamin A/C in the nucleoplasm (Fig. 6C-D).

To understand the relative contributions of assembled vs. soluble Lamin A/C to lamin mislocalization, we washed out soluble nuclear proteins in semi-permeabilized nuclei of healthy control and R249Q mutant iPSC-CMs, as a representative cell line. We imaged the remaining nonsoluble Lamin A/C (Fig. S7A) and quantified the un-normalized Lamin A/C fluorescence intensity in the nucleoplasm and at the NE to better capture the differences in protein levels at each location (Fig. S7B). Both R249Q mutant and healthy control iPSC-CMs had a similar pool of nucleoplasmic Lamin A/C that decreased with washout (Fig. S7B), indicating that much of the nucleoplasmic Lamin A/C was soluble and that R249Q mutant iPSC-CMs maintain a similar pool of nucleoplasmic Lamin A/C to healthy controls. However, the R249Q mutant iPSC-CMs had significantly decreased Lamin A/C at the NE compared to healthy controls, both in non-washout and washout conditions (Fig. S6B), suggesting that the R249Q mutation results in reduced Lamin $\mathrm{A} / \mathrm{C}$ assembly at the NE. Lamin A/C fluorescence intensity profiles for non-washout (Fig. S7C) and washout (Fig. S7D) iPSC-CMs provide additional visual confirmation of these results. Together, these results suggest that the changes in Lamin A/C localization can be attributed to reduced levels of Lamin $\mathrm{A} / \mathrm{C}$ assembled at the NE.

Lamin A/C and Lamin B1 form separate but interacting protein meshworks at the nuclear periphery (Shimi et al., 2015; Xie et al., 2016), and mutation or depletion of either protein can disrupt the structural organization of the other (Vigouroux et al., 2001; Muchir et al., 2004; Guo et al., 2014; Shimi et al., 2015; Steele-Stallard et al., 2018). Thus, we hypothesized that perturbed Lamin A/C assembly could also impact the organization of Lamin B1 in the LMNA mutant iPSCCMs. Indeed, all three $L M N A$ mutant iPSC-CM lines exhibited an increased nucleoplasmic to peripheral ratio of Lamin B1 compared to healthy control iPSC-CMs (Fig. 6E), with R249Q iPSCCMs exhibiting the most severe defects in Lamin B1 intranuclear distribution, and L35P and G449V iPSC-CMs displaying milder abnormalities. Collectively, these data indicate that mislocalization of Lamin A/C from the NE can alter Lamin B1 distribution. Furthermore, they suggest that the particularly severe defects in nuclear morphology and stability in the R249Q mutant iPSC-CMs could potentially arise in part from the mislocalization of Lamin B1 from nuclear periphery, given the critical role of B-type lamins in nuclear stability and preventing NE rupture (Lammerding et al., 2006; Hatch et al., 2013; Chen et al., 2018, 2019).

Lamin A/C phosphorylation is not altered in LMNA mutant iPSC-CMs 
An altered ratio of nucleoplasmic to peripheral Lamin $\mathrm{A} / \mathrm{C}$ could be due to a reduced ability of mutant Lamin A/C to self-assemble into higher order filaments (Wiesel et al., 2008; Zwerger et al., 2013; Bertrand et al., 2020) or an increase in Lamin A/C phosphorylation, which inhibits Lamin A/C's ability to form dimers and assemble at the nuclear periphery (Swift et al., 2013; Buxboim et al., 2014). To test whether increased phosphorylation of Lamin A/C was responsible for reduced Lamin $\mathrm{A} / \mathrm{C}$ localization to the NE, we immunofluorescently labeled iPSC-CMs for Lamin A/C phosphorylated at Serine 22 (p-Lamin A/C) and for total Lamin A/C (Fig. S8A). We did not detect any significant increase in the ratio of phosphorylated Lamin $\mathrm{A} / \mathrm{C}$ to total Lamin $\mathrm{A} / \mathrm{C}$ in the LMNA mutant iPSC-CMs (Fig. S8B), indicating that increased Lamin A/C phosphorylation is not responsible for reduced peripheral Lamin $\mathrm{A} / \mathrm{C}$ in these cells. These data suggest instead that likely altered self-assembly of Lamin $\mathrm{A} / \mathrm{C}$ into higher order filaments at the $\mathrm{NE}$ is responsible for lamin mislocalization in LMNA-mutant iPSC-CMs.

\section{Nuclear abnormalities correlate with lamin mislocalization from the nuclear envelope}

Our data suggest that $L M N A$ mutant iPSC-CMs have, to a varied degree, defects in Lamin $\mathrm{A} / \mathrm{C}$ and Lamin B1 localization to the nuclear periphery and that the R249Q mutant iPSC-CMs show particularly severe defects in both Lamin A/C and Lamin B1 organization, an observation which may explain the increased susceptibility of these cells to nuclear damage. To determine the degree to which mislocalization of lamins can explain nuclear size and shape defects in different cell lines, we computed a "lamin mislocalization index" from the average of the nucleoplasmic to peripheral Lamin A/C and Lamin B1 ratios for each cell line and correlated this index with a normalized "nuclear defects score," computed from the geometric mean of nuclear circularity index, area, and volume (Fig. 7). Intriguingly, the nuclear defects score across the panel of iPSCCMs strongly correlated with the lamin mislocalization index $\left(\mathrm{R}^{2}=0.96\right)$ with a significantly positive slope $(p=0.004)$, indicating that the extent of impaired Lamin $\mathrm{A} / \mathrm{C}$ and Lamin B1 assembly at the NE potentially explains the varying degrees of nuclear damage severities across multiple cell lines.

\section{Discussion}

The mechanisms through which different $L M N A$ mutations cause an array of disease phenotypes and severities has long remained an open question. Here, using three $L M N A$ mutant iPSC lines associated with $L M N A$-DCM, we made the novel discovery that the degree of impaired localization of Lamin A/C and Lamin B1 to the NE can explain much of the varying severities of nuclear damage observed in iPSC-CMs with different $L M N A$ mutations. We propose a mechanism in which $L M N A$ mutations cause defective assembly of Lamin $\mathrm{A} / \mathrm{C}$, resulting in reduced Lamin $\mathrm{A} / \mathrm{C}$ levels at the nuclear periphery, which, in turn, perturbs Lamin B1 localization to the nuclear periphery. Collectively, this reduced lamin assembly at the NE leads to impaired nuclear stability, resulting in defects in nuclear shape and size and increased nuclear fragility and NE rupture (Fig. 8).

Similar to iPSCs from healthy controls, $L M N A$ mutant iPSCs have low expression of Lamin $\mathrm{A} / \mathrm{C}$ and lack nuclear shape abnormalities. Upon differentiation into cardiomyocytes, Lamin A/C expression is upregulated, and LMNA mutant iPSC-CMs display varying degrees of nuclear abnormalities in the form of altered nuclear shape and/or increased nuclear size compared to healthy controls. R249Q mutant iPSC-CMs had the most severe nuclear size and shape defects and exhibited significantly more NE ruptures than the other iPSC-CMs, likely due to their reduced nuclear stiffness and stability. Healthy control iPSC-CMs depleted of Lamin A/C showed similar 
defects in nuclear morphology and increased NE rupture, as in the LMNA mutant iPSC-CMs, particularly the R249Q cells, confirming our observations in an isogenic background.

Intriguingly, R249Q mutant iPSC-CMs exhibited higher levels of NE rupture than the Lamin A/C-depleted iPSC-CMs. Furthermore, all LMNA mutant iPSC-CMs had essentially normal overall levels of Lamin A/C, although the R249Q iPSC-CMs showed a trend towards lower Lamin $\mathrm{A} / \mathrm{C}$ expression, which may cause some degree of $L M N A$ haploinsufficiency. These findings suggest that at least some $L M N A$ mutations function in a dominant negative manner. In support of this idea, expression of the R249Q equivalent in Drosophila Lamin C caused dominant effects on nuclear structure, including alterations in the organization of A- and B-type lamins (Hinz et al., 2021). Furthermore, in silico analysis of the Lamin A/C R249Q dimer predicted an arched conformation in the alpha helical rod domain of the extended Lamin $\mathrm{A} / \mathrm{C}$ model that could be responsible for altered lamina assembly (Hinz et al., 2021).

Pointing to a potential mechanism, all three $L M N A$ mutant iPSC-CM lines exhibited Lamin $\mathrm{A} / \mathrm{C}$ and Lamin B1 mislocalization from the nuclear periphery compared to healthy controls. This is likely due to defective assembly of Lamin $\mathrm{A} / \mathrm{C}$ at the nuclear lamina, as the $L M N A$ mutant cells still showed reduced Lamin A/C levels at the NE after removing soluble lamins (Fig. S7). These results are in line with previous studies showing that LMNA mutations may result in Lamin A/C mislocalization from the NE (Wiesel et al., 2008; Zwerger et al., 2013; Steele-Stallard et al., 2018; Bertrand et al., 2020), and that disrupting Lamin A/C assembly at the nuclear periphery impairs nuclear stability (Zwerger et al., 2015). However, although it is now well recognized that Lamin $\mathrm{A} / \mathrm{C}$ and Lamin B1 form interacting meshworks at the nuclear periphery and that depletion of one filament system disrupts and disrupts the structure of the other (Vigouroux et al., 2001; Muchir et al., 2004; Guo et al., 2014; Shimi et al., 2015; Steele-Stallard et al., 2018), little work has been done to understand the consequences of altered Lamin A/C assembly on Lamin B1 organization in laminopathies. The results presented here demonstrate that beyond disrupting the structure of Lamin B1, LMNA mutations can displace Lamin B1 from the NE, which is expected to make cells more susceptible to NE rupture (Lammerding et al., 2006; Hatch et al., 2013; Denais et al., 2016).

While previous studies have shown that Lamin A/C mislocalization correlates with defective nuclear shape (Wiesel et al., 2008; Zwerger et al., 2013; Steele-Stallard et al., 2018; Bertrand et al., 2020) and altered nuclear mechanics (Lammerding et al., 2004; Zwerger et al., 2015), our results point to an intriguing discovery that the 'lamin mislocalization index,' describing defects in both Lamin A/C and Lamin B1 assembly at the NE, can explain $>95 \%$ of the variability in nuclear defects in different $L M N A$ mutations and healthy control cell lines. Interestingly, at the level of individual nuclei, this correlation between lamin mislocalization and degree of nuclear abnormalities is roughly maintained among healthy control iPSC-CM nuclei but not R249Q nuclei (Fig. S9), suggesting that the increase in nuclear defects observed in R249Q mutant iPSC-CMs cannot be solely explained by an increase in lamin mislocalization from the nuclear periphery. This could potentially be due to a threshold for amount of lamin assembled at the NE, below which nuclei are increasingly susceptible to nuclear abnormalities. R249Q mutant iPSC-CMs generally have a high degree of both Lamin A/C and Lamin B1 mislocalization from the NE and evidence of significantly decreased nuclear stiffness, which could indicate that their nuclei are below this threshold for peripheral lamin assembly and thus are more susceptible to deformation. Additionally, we hypothesize that other changes to the cellular or nuclear structure, such as differences in contractility or force exertion on the NE (Cho et al., 2019), could drive increased nuclear abnormalities in this population. However, one limitation of this study is that iPSC-CMs were neither uniformly aligned on a substrate nor electrically paced, thus resulting in disorganized 
cellular structure with likely variation in cellular contractility. As actin contractility is known to play a role in nuclear damage (Hatch and Hetzer, 2016; Cho et al., 2019), it is plausible that the variability between individual nuclei within a cell line could be attributed at least in part to differences in contractility and force transmission to the NE: nuclei which are subject to increased amounts of force, or force that is applied to the nucleus in different ways, could increase nuclear abnormalities.

The loss of nuclear mechanical strength and NE integrity due to the loss of Lamin $\mathrm{A} / \mathrm{C}$ and/or Lamin B1 at the NE has drastic mechanical consequences on cellular health and survival (Denais et al., 2016; Chen et al., 2018, 2019; Cho et al., 2019; Earle et al., 2019; Shah et al., 2021), particularly through susceptibility of the nucleus to damage from intracellular forces (Hatch and Hetzer, 2016; Cho et al., 2019; Earle et al., 2019; Shah et al., 2021). However, one interesting observation of this study is that while the degree of lamin mislocalization works well to predict the effect of the $L M N A$ mutation on nuclear mechanics and stability, it is not necessarily a good predictor of patient phenotype. Of the three $L M N A$ mutations used in this study, the patient with the L35P mutation had the most severe phenotype, presenting both skeletal muscle and cardiac phenotypes during childhood and living only to age 15 . Conversely, the patient presenting the R249Q mutation, whose iPSC-CMs had more severe disruptions to nuclear mechanics than L35P iPSC-CMs, developed early onset muscular dystrophy in childhood, but only developed severe cardiac dysfunction later in life. This disparity between patient and cellular phenotype is not completely surprising, as additional defects caused by LMNA mutations, such as altered gene expression or biochemical signaling likely contribute to disease onset and progression. For example, altered Lamin A/C and Lamin B1 assembly at the NE can cause changes in chromatin organization and gene expression (Bertero et al., 2019; Cheedipudi et al., 2019; Kim et al., 2019), including nuclear mechanotransduction and associated signaling (Kirby and Lammerding, 2018; Maurer and Lammerding, 2019; Donnaloja et al., 2020). As such, loss of assembled Lamin A/C and/or Lamin B1 at the nuclear periphery may impair mechanically-induced gene expression (Maurer and Lammerding, 2019; Donnaloja et al., 2020).

Overall, our results point to the degree of lamin mislocalization from the NE as an important mechanism across LMNA-DCM mutations which determines the degree of nuclear mechanical damage in cardiomyocytes. Moreover, altered lamin assembly at the NE may present a potential link between the proposed laminopathy pathogenic mechanisms of impaired nuclear stability and altered gene expression driving LMNA mutant skeletal muscle and cardiac laminopathies (Maurer and Lammerding, 2019; Donnaloja et al., 2020). Future studies should be aimed at determining the molecular mechanism(s) by which nuclear damage causes cardiac dysfunction in $L M N A$-DCM, including obtaining a better understanding of changes in mechanotransduction signaling and gene expression. Additionally, our work and others showing that Lamin $\mathrm{A} / \mathrm{C}$ assembly has implications in the degree of nuclear damage and altered nuclear mechanics (Wiesel et al., 2008; Zwerger et al., 2013; Bertrand et al., 2020) suggest that by understanding the mutation-specific degree of defective lamin assembly, we may ultimately be able to identify patient mutations that would most benefit from therapeutics targeting the reduction of force transmission to the nucleus (Cho et al., 2019; Earle et al., 2019; Chai et al., 2021). Although additional mechanisms may drive $L M N A$-DCM, such therapeutics hold promise to improve cellular health and survival (Cho et al., 2019; Earle et al., 2019; Chai et al., 2021).

\section{Acknowledgements}


The authors thank the Cure Muscular Dystrophy Foundation for the LMNA L35P iPSCs; Hanna Gimse for analyzing the nuclear volume in shRNA experiments; the Cornell Institute of Biotechnology for performing library preparation and Illumina sequencing experiments; the Cornell Statistical Consulting Unit for developing the regressions performed in R; Dr. Kehan Zhang and Dr. Christopher Chen for hosting M.E.M. to learn iPSC culture and cardiac differentiation protocols; and Dr. Kathleen Xie for the nuclear semi-permeabilization protocol. This work was supported by awards from the National Institutes of Health (R01 HL082792 to J.L., R01 HL128075 to E.M.M., and F30 HL142187 to A.M.G.); the National Science Foundation (CBET 1715606 to J.L.; Graduate Research Fellowships 2016229710 to M.M.), the American Heart Association (20PRE35080179 to M.M.), the Muscular Dystrophy Association (MDA603238 to T.J.K), and the Volkswagen Foundation (A130142 to J.L.).

\section{References}

Andrew, S (2010). FastQC: A Quality Control Tool for High Throughput Sequence Data [Online].

Bertero, A et al. (2019). Chromatin compartment dynamics in a haploinsufficient model of cardiac laminopathy. J Cell Biol 218, 2919-2944.

Bertrand, AT, Brull, A, Azibani, F, Benarroch, L, Chikhaoui, K, Stewart, CL, Medalia, O, Ben Yaou, R, and Bonne, G (2020). Lamin A/C Assembly Defects in LMNA-Congenital Muscular Dystrophy Is Responsible for the Increased Severity of the Disease Compared with Emery-Dreifuss Muscular Dystrophy. Cells 9, 844.

Bolte, S, and Cordelières, F (2006). A guided tour into subcellular colocalization analysis in light microscopy. J Microsc 224, 213-232.

Broers, JLV, Peeters, EAG, Kuijpers, HJH, Endert, J, Bouten, CVC, Oomens, CWJ, Baaijens, FPT, and Ramaekers, FCS (2004). Decreased mechanical stiffness in LMNA-/- cells is caused by defective nucleo-cytoskeletal integrity: Implications for the development of laminopathies. Hum Mol Genet 13, 2567-2580.

Buxboim, A et al. (2014). Matrix Elasticity Regulates Lamin-A,C Phosphorylation and Turnover with Feedback to Actomyosin. Curr Biol 24, 1909-1917.

Cattin, ME et al. (2013). Heterozygous LmnadelK32 mice develop dilated cardiomyopathy through a combined pathomechanism of haploinsufficiency and peptide toxicity. Hum Mol Genet 22, 3152-3164.

Cenni, V et al. (2005). Lamin A N-terminal phosphorylation is associated with myoblast activation: impairment in Emery-Dreifuss muscular dystrophy. J Med Genet 42, 214-220.

Chai, RJ et al. (2021). Disrupting the LINC complex by AAV mediated gene transduction prevents progression of Lamin induced cardiomyopathy. Nat Commun 12, 1-16.

Chandar, $\mathrm{S}$ et al. (2010). Effects of mechanical stress and carvedilol in Lamin A/C-deficient dilated cardiomyopathy. Circ Res 106, 573-582.

Cheedipudi, SM et al. (2019). Genomic Reorganization of Lamin-Associated Domains in Cardiac Myocytes is Associated with Differential Gene Expression and DNA Methylation in Human Dilated Cardiomyopathy. Circ Res 124, 1198.

Chen, NY et al. (2019). An absence of Lamin B1 in migrating neurons causes nuclear membrane ruptures and cell death. Proc Natl Acad Sci, 201917225.

Chen, NY, Kim, P, Weston, TA, Edillo, L, Tu, Y, Fong, LG, and Young, SG (2018). Fibroblasts 
lacking nuclear lamins do not have nuclear blebs or protrusions but nevertheless have frequent nuclear membrane ruptures. Proc Natl Acad Sci, 201812622.

Cho, S et al. (2019). Mechanosensing by the Lamina Protects against Nuclear Rupture, DNA Damage, and Cell-Cycle Arrest. Dev Cell.

Davidson, PM, and Lammerding, J (2014). Broken nuclei - lamins, nuclear mechanics, and disease. Trends Cell Biol 24, 247-256.

Denais, CM, Gilbert, RM, Isermann, P, McGregor, AL, Te Lindert, M, Weigelin, B, Davidson, PM, Friedl, P, Wolf, K, and Lammerding, J (2016). Nuclear envelope rupture and repair during cancer cell migration. Science (80- ).

Dobin, A, Davis, CA, Schlesinger, F, Drenkow, J, Zaleski, C, Jha, S, Batut, P, Chaisson, M, and Gingeras, TR (2013). STAR: Ultrafast universal RNA-seq aligner. Bioinformatics 29, 15-21.

Donnaloja, F, Carnevali, F, Jacchetti, E, and Raimondi, MT (2020). Lamin A/C Mechanotransduction in Laminopathies. Cells 9, 1306.

Earle, AJ, Kirby, TJ, Fedorchak, GR, Isermann, P, Patel, J, Iruvanti, S, Moore, SA, Bonne, G, Wallrath, LL, and Lammerding, J (2019). Mutant lamins cause nuclear envelope rupture and DNA damage in skeletal muscle cells. Nat Mater 19, 464-473.

Guo, Y, Kim, Y, Shimi, T, Goldman, RD, and Zheng, Y (2014). Concentration-dependent lamin assembly and its roles in the localization of other nuclear proteins. Mol Biol Cell 25, 12871297.

Gupta, P et al. (2010). Genetic and ultrastructural studies in dilated cardiomyopathy patients: a large deletion in the Lamin $\mathrm{A} / \mathrm{C}$ gene is associated with cardiomyocyte nuclear envelope disruption. Basic Res Cardiol 105, 365-377.

Guz, N, Dokukin, M, Kalaparthi, V, and Sokolov, I (2014). If Cell Mechanics Can Be Described by Elastic Modulus: Study of Different Models and Probes Used in Indentation Experiments. Biophys J 107, 564-575.

Halfmann, CT, Sears, RM, Katiyar, A, Busselman, BW, Aman, LK, Zhang, Q, O'Bryan, CS, Angelini, TE, Lele, TP, and Roux, KJ (2019). Repair of nuclear ruptures requires barrier-toautointegration factor. J Cell Biol, jcb.201901116.

Hasselberg, NE, Haland, TF, Saberniak, J, Brekke, PH, Berge, KE, Leren, TP, Edvardsen, T, and Haugaa, KH (2018). Lamin A/C cardiomyopathy: Young onset, high penetrance, and frequent need for heart transplantation. Eur Heart J 39, 853-860.

Hatch, EM, Fischer, AH, Deerinck, TJ, and Hetzer, MW (2013). Catastrophic Nuclear Envelope Collapse in Cancer Cell Micronuclei. Cell 154, 47-60.

Hatch, EM, and Hetzer, MW (2016). Nuclear envelope rupture is induced by actin-based nucleus confinement. J Cell Biol 215, 27-36.

Heffler, J, Shah, PP, Robison, P, Phyo, S, Veliz, K, Uchida, K, Bogush, A, Rhoades, J, Jain, R, and Prosser, BL (2019). A Balance Between Intermediate Filaments and Microtubules Maintains Nuclear Architecture in the Cardiomyocyte. Circ Res.

Hinz, BE, Walker, SG, Xiong, A, Gogal, RA, Schnieders, MJ, and Wallrath, LL (2021). In Silico and In Vivo Analysis of Amino Acid Substitutions That Cause Laminopathies. Int J Mol Sci 2021, Vol 22, Page 11226 22, 11226.

Kim, Y, Zheng, X, and Zheng, Y (2019). Role of lamins in 3D genome organization and global gene expression. Https://DoiOrg/101080/1949103420191578601 10, $13-21$.

Kirby, TJ, and Lammerding, J (2018). Emerging views of the nucleus as a cellular mechanosensor. Nat Cell Biol 20, 373-381.

Lammerding, J, Fong, LG, Ji, JY, Reue, K, Stewart, CL, Young, SG, and Lee, RT (2006). 
Lamins A and C but not Lamin B1 regulate nuclear mechanics. J Biol Chem 281, 2576825780.

Lammerding, J, Schulze, PC, Takahashi, T, Kozlov, S, Sullivan, T, Kamm, RD, Stewart, CL, and Lee, RT (2004). Lamin A/C deficiency causes defective nuclear mechanics and mechanotransduction. J Clin Invest 113, 370-378.

Liao, Y, Smyth, GK, and Shi, W (2014). FeatureCounts: An efficient general purpose program for assigning sequence reads to genomic features. Bioinformatics 30, 923-930.

Love, MI, Huber, W, and Anders, S (2014). Moderated estimation of fold change and dispersion for RNA-seq data with DESeq2. Genome Biol 15, 550.

Maurer, M, and Lammerding, J (2019). The Driving Force: Nuclear Mechanotransduction in Cellular Function, Fate, and Disease. Annu Rev Biomed Eng 21, 443-468.

McNally, EM, and Mestroni, L (2017). Dilated cardiomyopathy: Genetic determinants and mechanisms. Circ Res 121, 731-748.

Muchir, A et al. (2004). Nuclear envelope alterations in fibroblasts from patients with muscular dystrophy, cardiomyopathy, and partial lipodystrophy carrying Lamin A/C gene mutations. Muscle Nerve 30, 444-450.

Nikolova, V et al. (2004). Defects in nuclear structure and function promote dilated cardiomyopathy in lamin A / C - deficient mice. J Clin Invest 113, 357-369.

Ollion, J, Cochennec, J, Loll, F, Escudé, C, and Boudier, T (2013). TANGO: a generic tool for high-throughput 3D image analysis for studying nuclear organization. Bioinformatics 29, $1840-1841$.

Piccus, R, and Brayson, D (2020). The nuclear envelope: LINCing tissue mechanics to genome regulation in cardiac and skeletal muscle. Biol Lett 16, 20200302.

Shah, D et al. (2019). Modeling of LMNA-Related Dilated Cardiomyopathy Using Human Induced Pluripotent Stem Cells. Cells 8, 594.

Shah, P, Hobson, CM, Cheng, S, Colville, MJ, Paszek, MJ, Superfine, R, and Lammerding, J (2021). Nuclear Deformation Causes DNA Damage by Increasing Replication Stress. Curr Biol 31, 753-765.e6.

Sharma, A, Li, G, Rajarajan, K, Hamaguchi, R, Burridge, PW, and Wu, SM (2015). Derivation of Highly Purified Cardiomyocytes from Human Induced Pluripotent Stem Cells Using Small Molecule-modulated Differentiation and Subsequent Glucose Starvation. J Vis Exp, 310.

Shimi, T, Kittisopikul, M, Tran, J, Goldman, AE, Adam, SA, Zheng, Y, Jaqaman, K, and Goldman, RD (2015). Structural organization of nuclear lamins A, C, B1, and B2 revealed by superresolution microscopy. Mol Biol Cell 26, 4075-4086.

Siu, CW et al. (2012). Modeling of Lamin A/C mutation premature cardiac aging using patientspecific induced pluripotent stem cells. Aging (Albany NY) 4, 803-822.

Steele-Stallard, HB, Pinton, L, Sarcar, S, Ozdemir, T, Maffioletti, SM, Zammit, PS, and Tedesco, FS (2018). Modeling Skeletal Muscle Laminopathies Using Human Induced Pluripotent Stem Cells Carrying Pathogenic LMNA Mutations. Front Physiol 9, 1332.

Stephens, AD, Banigan, EJ, Adam, SA, Goldman, RD, and Marko, JF (2017). Chromatin and lamin A determine two different mechanical response regimes of the cell nucleus. Mol Biol Cell 28, 1984-1996.

Stewart, CL, Kozlov, S, Fong, LG, and Young, SG (2007). Mouse models of the laminopathies. Exp Cell Res 313, 2144-2156.

Sullivan, T, Escalante-Alcalde, D, Bhatt, H, Anver, M, Bhat, N, Nagashima, K, Stewart, CL, and 
Burke, B (1999). Loss of A-type lamin expression compromises nuclear envelope integrity leading to muscular dystrophy. J Cell Biol 147, 913-920.

Swift, J et al. (2013). Nuclear Lamin-A Scales with Tissue Stiffness and Enhances MatrixDirected Differentiation. Science (80- ) 341.

Taylor, MRG et al. (2003). Natural history of dilated cardiomyopathy due to Lamin A/C gene mutations. J Am Coll Cardiol 41, 771-780.

Vigouroux, C, Auclair, M, Dubosclard, E, Pouchelet, M, Capeau, J, Courvalin, JC, and Buendia, B (2001). Nuclear envelope disorganization in fibroblasts from lipodystrophic patients with heterozygous R482Q/W mutations in the Lamin A/C gene. J Cell Sci 114, 4459-4468.

De Vos, WH et al. (2011). Repetitive disruptions of the nuclear envelope invoke temporary loss of cellular compartmentalization in laminopathies. Hum Mol Genet 20, 4175-4186.

Wiesel, N, Mattout, A, Melcer, S, Melamed-Book, N, Herrmann, H, Medalia, O, Aebi, U, and Gruenbaum, Y (2008). Laminopathic mutations interfere with the assembly, localization, and dynamics of nuclear lamins. Proc Natl Acad Sci U S A 105, 180-185.

Wolf, CM et al. (2008). Lamin A/C haploinsufficiency causes dilated cardiomyopathy and apoptosis-triggered cardiac conduction system disease. J Mol Cell Cardiol 44, 293-303.

Xie, W, Chojnowski, A, Boudier, T, Lim, JSY, Ahmed, S, Ser, Z, Stewart, C, and Burke, B (2016). A-type Lamins Form Distinct Filamentous Networks with Differential Nuclear Pore Complex Associations. Curr Biol 26, 2651-2658.

Zuo, B, Yang, J, Wang, F, Wang, L, Yin, Y, Dan, J, Liu, N, and Liu, L (2012). Influences of lamin A levels on induction of pluripotent stem cells. Biol Open 1, 1118-1127.

Zwerger, M, Jaalouk, DE, Lombardi, ML, Isermann, P, Mauermann, M, Dialynas, G, Herrmann, H, Wallrath, L, and Lammerding, J (2013). Myopathic lamin mutations impair nuclear stability in cells and tissue and disrupt nucleo-cytoskeletal coupling. Hum Mol Genet 22, 2335-2349.

Zwerger, M, Roschitzki-voser, H, Zbinden, R, Denais, C, and Herrmann, H (2015). Altering lamina assembly identifies lamina-dependent and -independent functions for A-type lamins Journal of Cell Science Accepted manuscript. J Cell Sci 41. 


\section{$\underline{\operatorname{Table}(\mathbf{s})}$}

\begin{tabular}{|c|c|c|c|c|c|c|}
\hline Protein & cDNA & Exon & $\begin{array}{l}\text { Protein } \\
\text { Domain }\end{array}$ & $\begin{array}{c}\text { Mutation } \\
\text { Origin }\end{array}$ & Affected Tissue & Cell Source \\
\hline p.L35P & c.T104C & 1 & Coil 1A & Unknown & $\begin{array}{c}\text { Skeletal and } \\
\text { cardiac muscle }\end{array}$ & $\begin{array}{l}\text { Cure CMD and } \\
\text { Cellular Dynamics } \\
\text { International Inc. }\end{array}$ \\
\hline p.R249Q & c.G746A & 1 & Coil 2 & De novo & $\begin{array}{c}\text { Skeletal and } \\
\text { cardiac muscle }\end{array}$ & Elizabeth McNally \\
\hline p.G449V & c.G1346T & 7 & Ig-fold & De novo & Skeletal muscle* & Ivor Benjamin \\
\hline
\end{tabular}

Table 1. Overview of $L M N A$-mutant iPSC-CM lines. The iPSC lines were isolated from patients carrying $L M N A$ mutations that produce amino acid substitutions in different domains of the Lamin $\mathrm{A} / \mathrm{C}$ protein, as indicated in the table, and cause either Congenital Muscular Dystrophy (CMD) or $L M N A$-DCM. The CMD patients with the L35P and G449V amino acid substitutions are expected to develop a cardiac phenotype later in life, in addition to the skeletal muscular dystrophy present at diagnosis. *The G449V patient presented with muscular dystrophy in childhood, but patient is too young to necessarily develop cardiac phenotype. 


\section{Figures \& Legends}
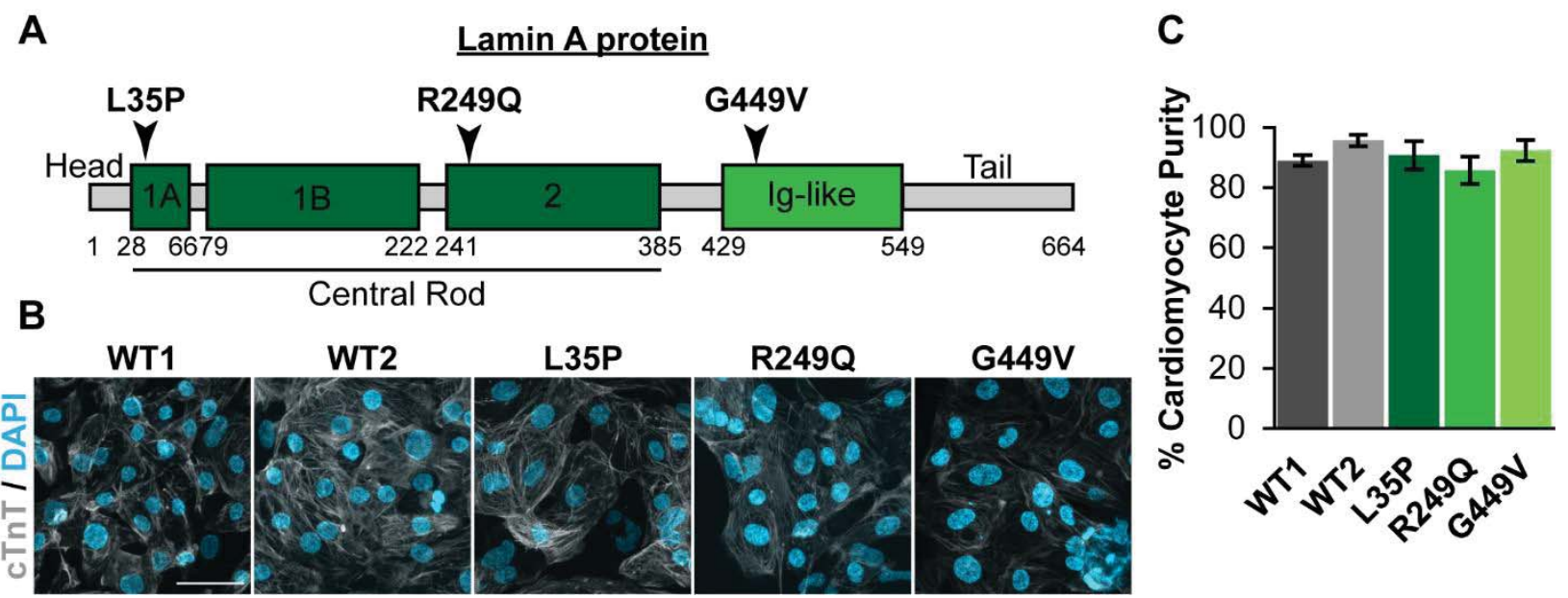

Figure 1. $L M N A$ mutant iPSCs differentiate into cardiomyocytes with high efficiency. (A) Diagram of Lamin A protein domains with the locations of the amino acid substitutions used in this study indicated, which map to separate domains of the Lamin A protein. Note that these domains are also present the Lamin $\mathrm{C}$ protein, which has an alternative tail domain compared to Lamin A. (B) Immunofluorescence images of iPSC-CMs show expression of the cardiac marker Cardiac Troponin T (cTnT) in the vast majority of cells. Scale bar $=50 \mu \mathrm{m}$. (C) Quantification of iPSC-CM purity based on cTnT labeling shows that all cell lines differentiate into cardiac myocytes with $>85 \%$ efficiency, with no statistically significant differences between the cell lines. Data represented as mean \pm SEM. $N=8$-14 images per group. 

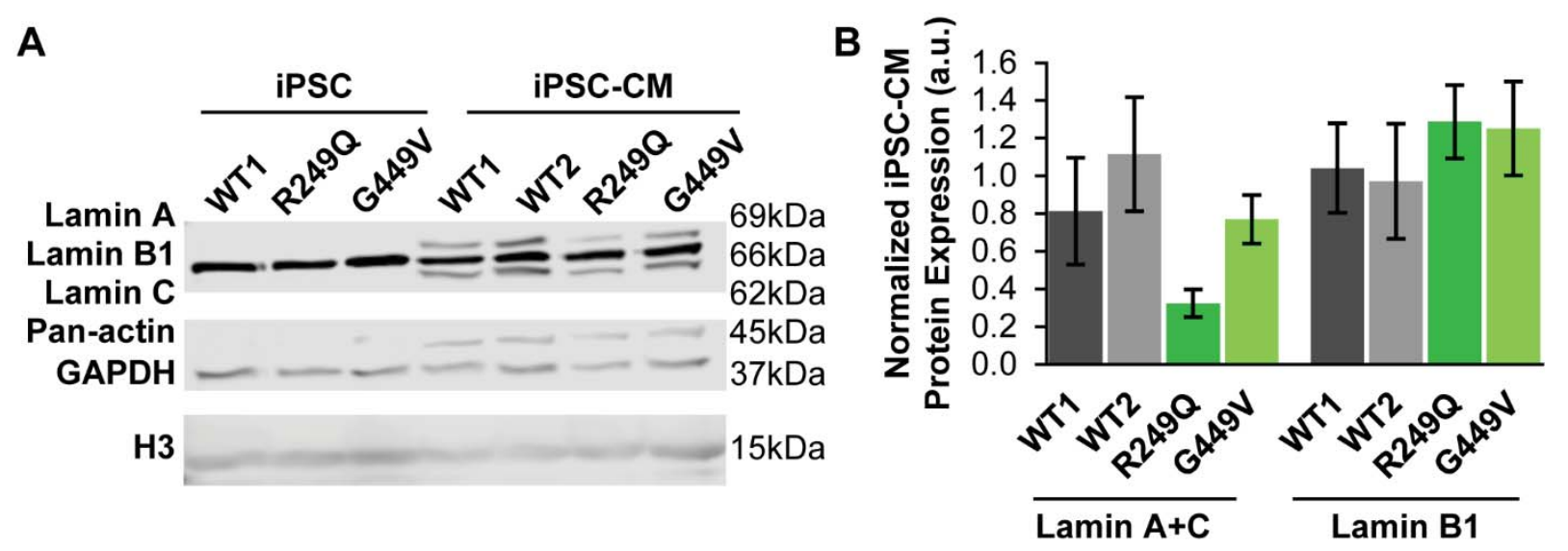

Figure 2. R249Q iPSC-CMs trend toward reduced Lamin A/C protein expression. (A) Representative western analysis, showing that iPSCs do not express detectable Lamin A/C, and that Lamin A/C expression increases in iPSC-CMs. (B) Quantification of lamin protein levels from western analysis of iPSC-CM samples, showing that G449V iPSC-CMs have normal levels of Lamin A/C, whereas R249Q iPSC-CMs have a trend towards decreased Lamin A/C protein levels that does not reach statistical significance ( $p>0.15$ vs both WT lines). Data presented as mean \pm SEM. $N=5$ protein lysates per group. 

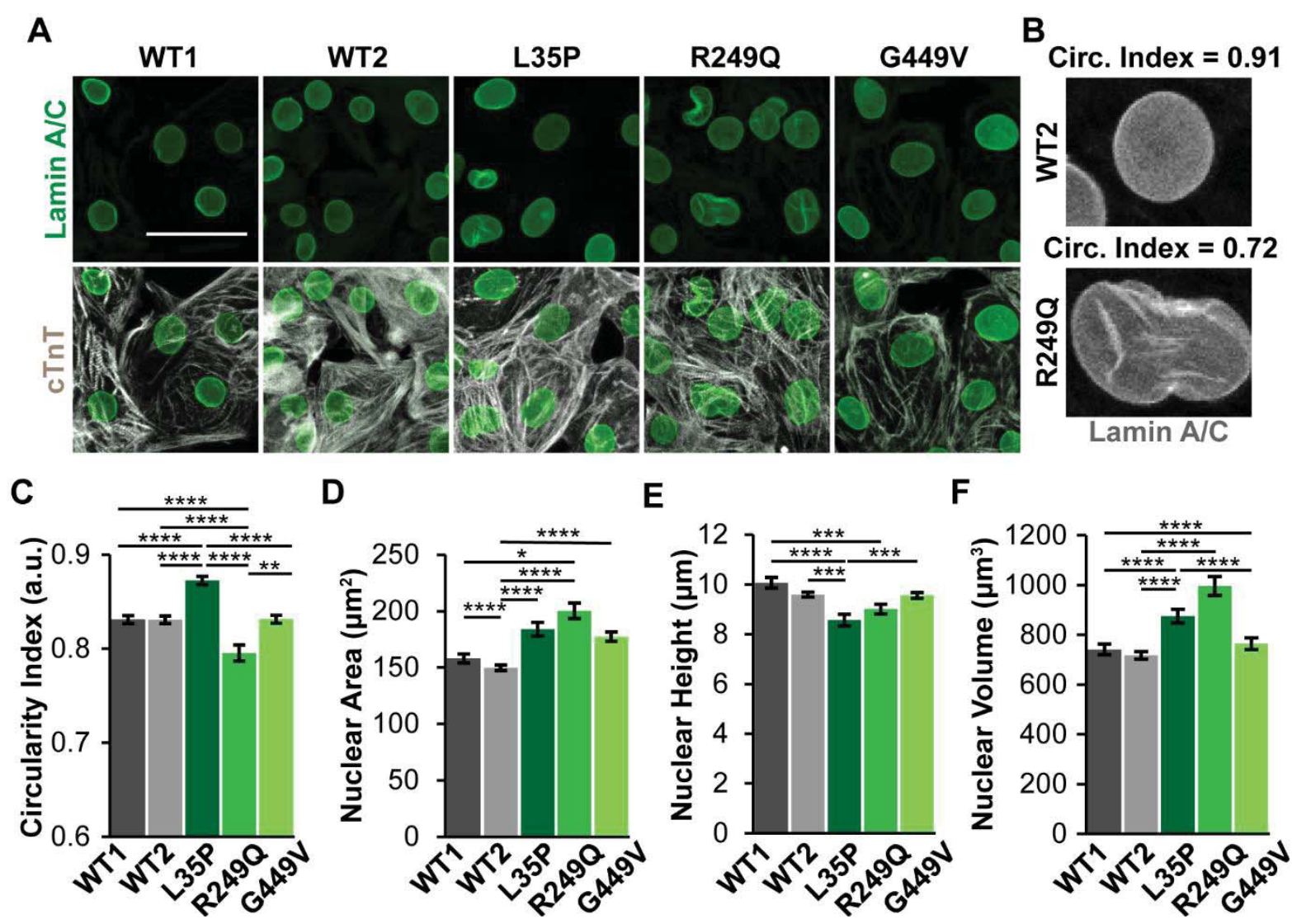

D

$\mathrm{E}$
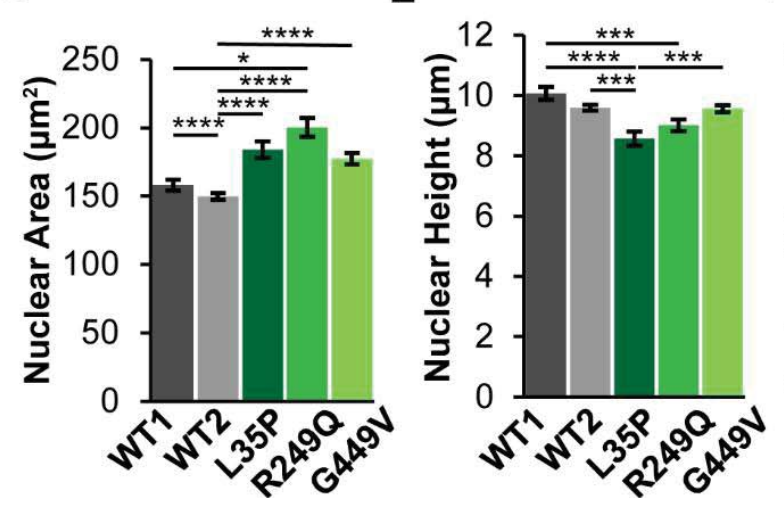

$\mathbf{F}$

Figure 3. LMNA mutant iPSC-CMs have nuclear shape and size defects. (A) Representative immunofluorescence images of iPSC-CMs stained for Lamin A/C (green) and cardiac troponin T (cTnT, gray). Scalebar $=50 \mu \mathrm{m}$. (B) Examples of WT2 and R249Q nuclei with corresponding circularity index. (C) R249Q nuclei have significantly decreased circularity index values compared to the WT cell lines, while L35P and G449V nuclei have an increase or no change in circularity index, respectively. (D) All LMNA-mutant iPSC-CMs exhibit an increase in nuclear area, but (E) only L35P and R249Q cells have decreased nuclear height. (F) L35P and R249Q iPSC-CMs have increased nuclear volume. Data presented as mean $\pm \mathrm{SEM} .{ }^{*}, p<0.05$ vs. WT, $* * *, p<0.001$ vs. WT, $* * * *, p<0.0001$ vs. WT. $N \geq 83$ nuclei per group for all experiments. 

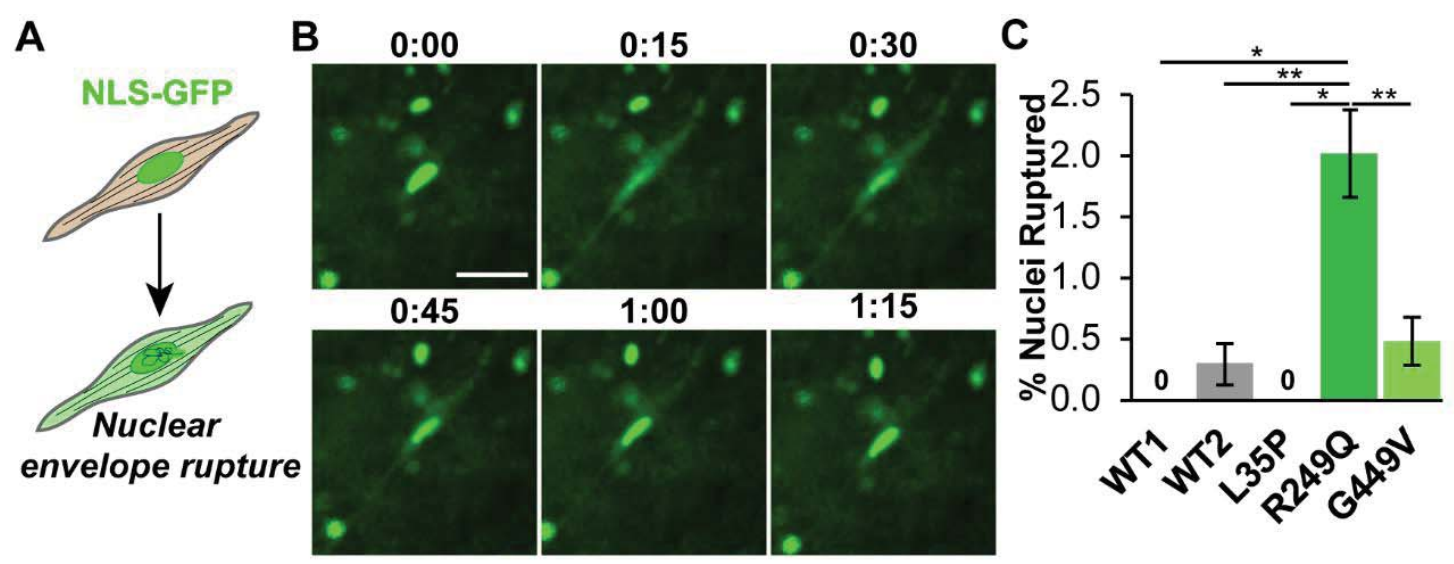

D

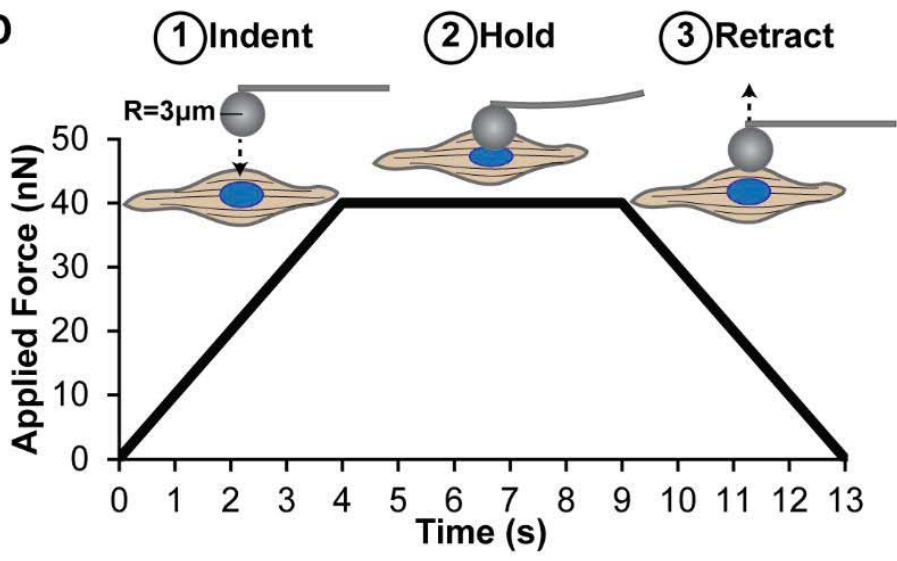

E

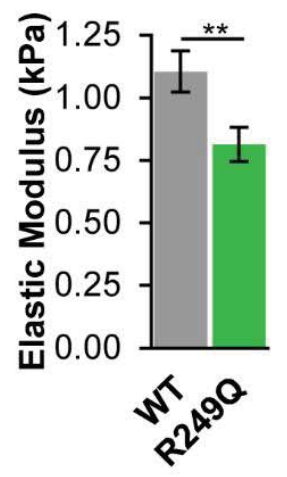

Figure 4. R249Q iPSC-CMs exhibit decreased nuclear stiffness and increased nuclear envelope rupture. (A) iPSC-CMs were modified with a nuclear envelope (NE) rupture reporter, NLS-GFP, consisting of GFP with a Nuclear Localization Sequence. NLS-GFP is normally packaged in the nucleus and leaks into the cytoplasm upon NE rupture. (B) Representative time lapse series of a G449V iPSC-CM undergoing NE rupture and repair. Scalebar $=30 \mu \mathrm{m}$. Time units are represented in hours:minutes. (C) Quantification of NE ruptures shows that R249Q iPSCCMs have significantly increased NE rupture compared to the healthy control (WT) iPSC-CM lines. (D) iPSC-CM nuclei were indented using a spherical probe on an Optics11 Chiaro nanoindenter to quantify nuclear stiffness. The indentation probe was lowered until it reached a load of $40 \mathrm{nN}$; the load was held for 5 seconds and then the probe was slowly retracted. (E) Quantification of nuclear elastic modulus, determined by fitting a Hertzian model to the loadindentation curves, revealed that R249Q nuclei are significantly softer compared to healthy control (WT) nuclei. NE rupture data presented as mean \pm SEP, nuclear stiffness data represented as mean \pm SEM. ${ }^{*}, p<0.05$ vs. WT, ${ }^{*}, p<0.01$ vs. WT. $N \geq 68$ nuclei per group for nuclear stiffness, $N$ $\geq 1020$ nuclei per group for NE rupture. 
A

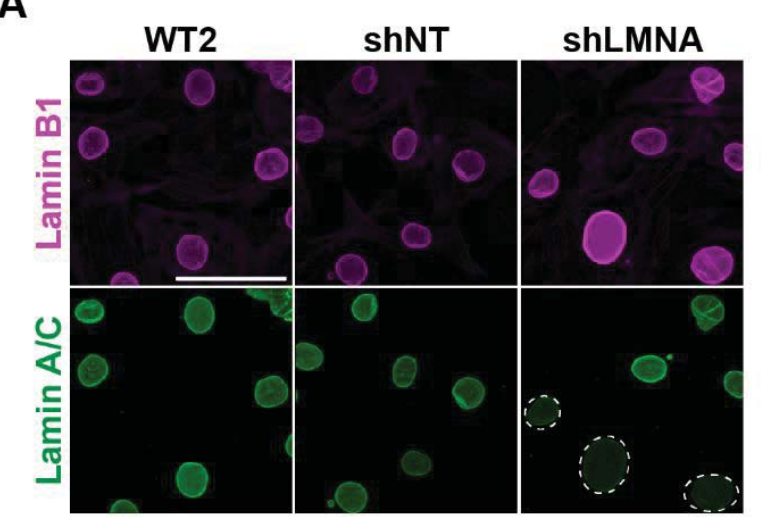

C

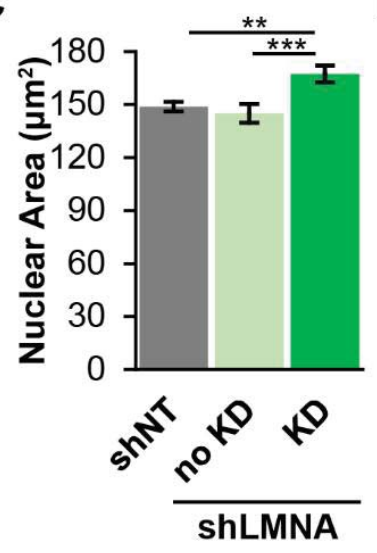

D

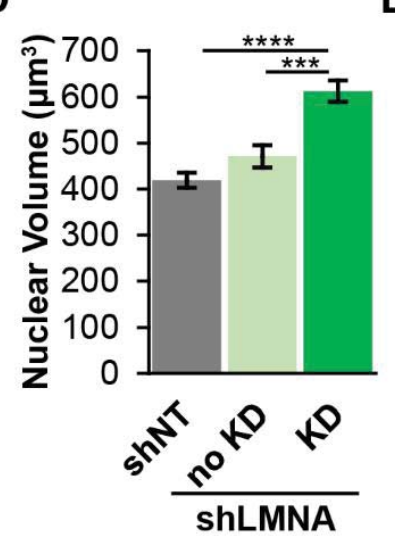

B

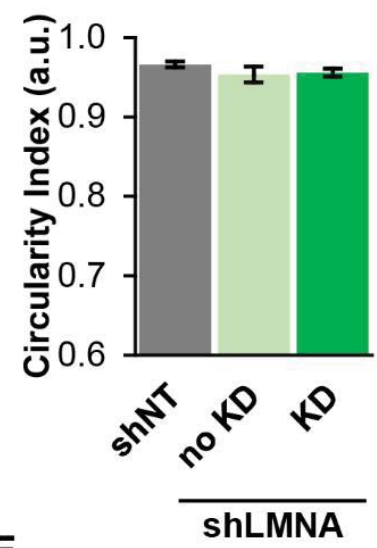

E

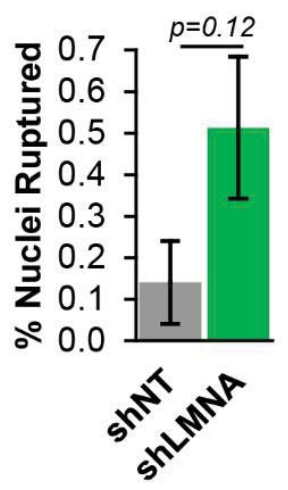

Figure 5. Depletion of Lamin A/C in healthy control iPSC-CMs causes increased nuclear area, volume, and nuclear envelope ruptures. (A) Representative immunofluorescence images for Lamin B1 and Lamin A/C in healthy control (WT) iPSC-CMs either modified with shRNA targeting LMNA (shLMNA) or a non-target control shRNA (shNT). White dotted circles mark shLMNA treated nuclei with visible Lamin A/C depletion. Scalebar $=50 \mu \mathrm{m}$. (B) shLMNA nuclei with depletion of Lamin A/C (knockdown; KD shLMNA) have no change in circularity index compared to both non-target (shNT) control and non-depleted shLMNA nuclei (no KD). (C) KD shLMNA nuclei have significantly increased nuclear area compared to both non-target (shNT) control and non-knockdown shLMNA nuclei. (D) KD shLMNA nuclei have significantly increased nuclear volume compared to both non-target (shNT) control and non-knockdown shLMNA nuclei. (E) Unselected populations of shLMNA treated iPSC-CMs had a trend towards increased NE rupture compared to shNT treated controls. Due to the experimental protocol using live cells, we could not distinguish cells with successful Lamin A/C depletion from those without Lamin A/C depletion in the shLMNA treated cells. Data presented as mean \pm SEM. ${ }^{* *}, p<0.01$ vs. control, ***, $p<0.001$ vs. control, $* * * *, p<0.0001$ vs. control. $N \geq 83$ nuclei per group for nuclear shape and area, $N \geq 47$ nuclei per group for nuclear volume, $N>1,415$ nuclei per group for NE rupture. 
A

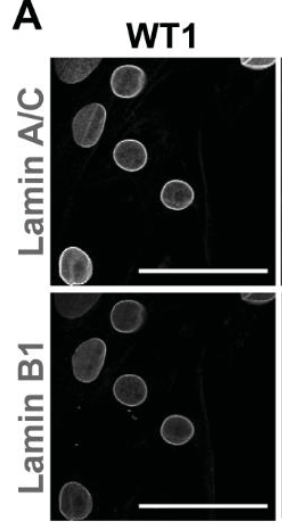

C

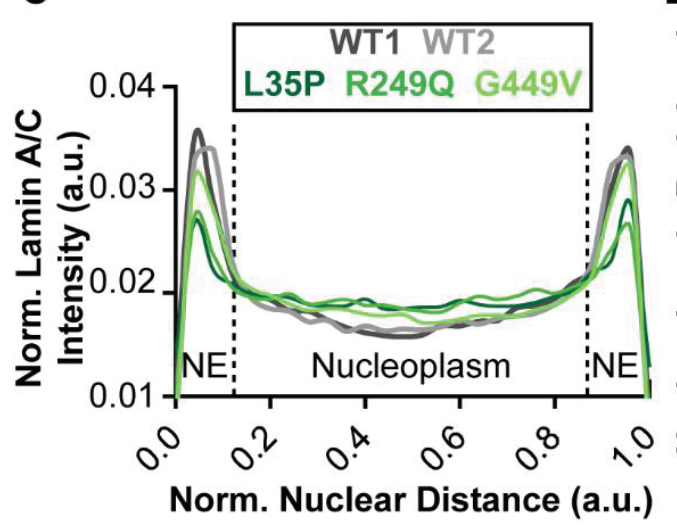

L35P
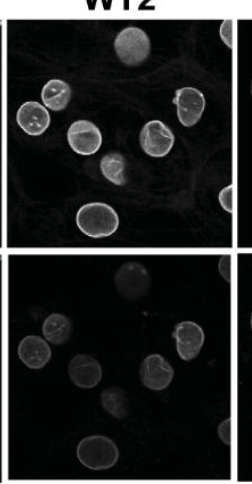

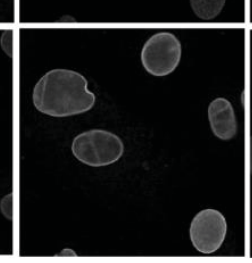

D

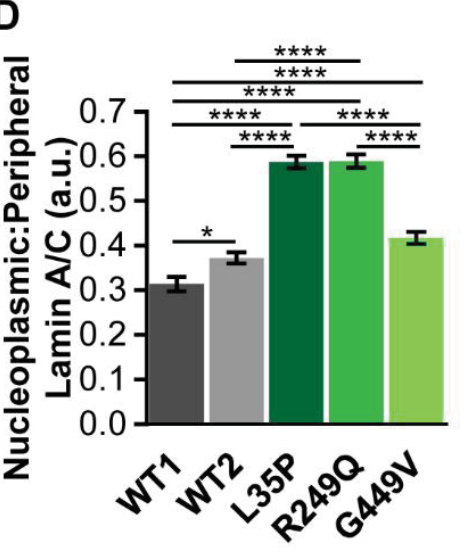

B

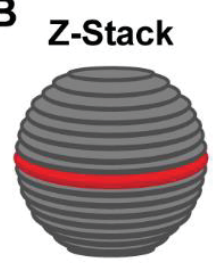

Lamin A/C

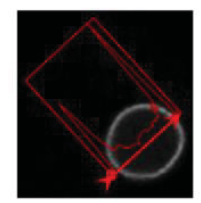

$\mathbf{E}$

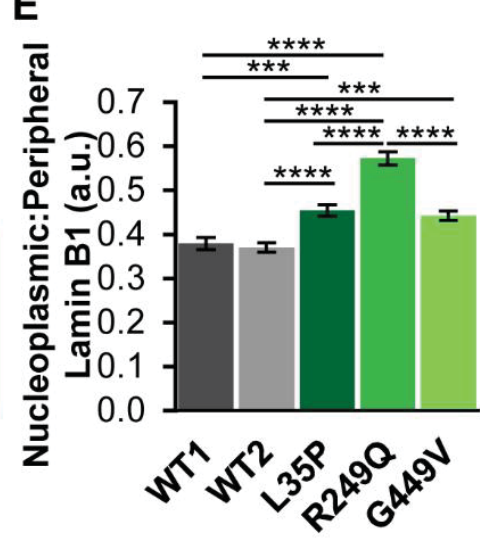

Figure 6. $L M N A$-mutant iPSC-CMs have mislocalization of Lamin A/C and Lamin B1 from the nuclear envelope. (A) Confocal immunofluorescence images of cross sections through the center of nuclei show that L35P and R249Q iPSC-CM nuclei have reduced fluorescence intensity of Lamin A/C at the nuclear periphery, and R249Q nuclei additionally have reduced fluorescence intensity of Lamin B1 at the nuclear periphery. Scalebars $=50 \mu \mathrm{m}$. (B) Schematic of the lamin localization profile analysis. Lamin A/C fluorescence intensity profiles were taken at the central z-plane of nuclei from confocal image slices. (C) A plot of the normalized Lamin A/C fluorescence intensity profile shows that L35P and R249Q nuclei have reduced fluorescence intensity peaks at the nuclear periphery (nuclear envelope; NE) and increased fluorescence intensity in the nucleoplasm. (D) L35P, R249Q, and G449V iPSC-CMs have a significantly increased ratio of nucleoplasmic to peripheral Lamin $\mathrm{A} / \mathrm{C}$, indicating lamin mislocalization away from the nuclear envelope. (E) L35P, R249Q, and G449V-iPSC-CMs have a significantly increased ratio of nucleoplasmic to peripheral Lamin B1, with R249Q nuclei having a particularly increased ratio, indicating lamin mislocalization away from the nuclear envelope. Data presented as mean $\pm \mathrm{SEM}$. $*, p<0.05$ vs. WT, ${ }^{* * *}, p<0.001$ vs. WT, $* * * *, p<0.0001$ vs. WT. $N>60$ nuclei per group. 


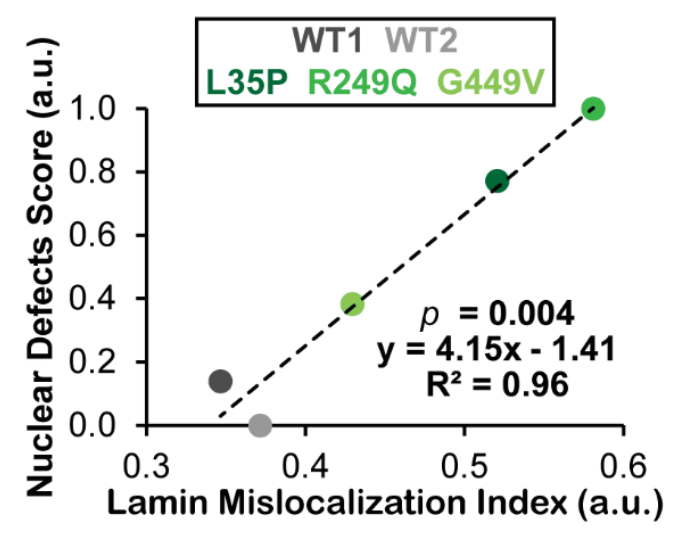

Figure 7. Lamin mislocalization correlates to nuclear damage in $L M N A$-mutant iPSC-CMs. Lamin mislocalization index (defined as the average of Lamin A/C and Lamin B1 nucleoplasmic to peripheral intensity ratios) shows a significant positive correlation to the nuclear defects score (defined as the normalized geometric mean of nuclear circularity index, area, and volume) for the five iPSC-CM cell lines used in this study. 


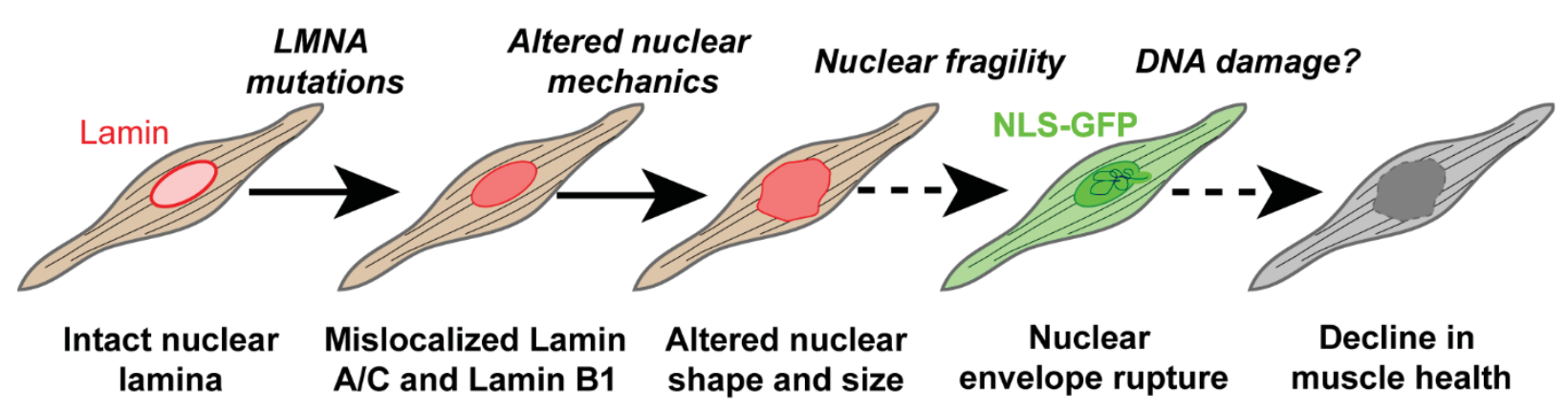

Figure 8. Proposed mechanism of nuclear damage in LMNA-mutant iPSC-CMs. Reduced Lamin A/C levels and/or defective assembly of Lamin A/C causes Lamin A/C mislocalization from the nuclear envelope. This mislocalization alters nuclear mechanics, particularly reducing nuclear stiffness and stability, leaving nuclei susceptible to cytoskeletal forces exerted on the nucleus in cardiac myocytes. These forces in turn cause abnormal nuclear size and shape and increased nuclear envelope rupture. Nuclear envelope rupture, and potentially other nuclear damage, can cause a decline in muscle health, potentially through mechanisms such as DNA damage or increased apoptosis. 